\title{
Una perspectiva ambiental en el planeamiento de la ferrovía bioceánica: modelamiento cuantitativo de escenarios
}

En octubre de 2015, China Railway Eryuan Engineering Group Co. LTD, desarrolló un estudio técnico para la construcción de la ferrovía bioceánica Perú - Brasil. E estudio propuso la ruta norte como la alternativa mejor viable. Sin embargo, esta alternativa fue criticada puesto que el proyecto colocó en riesgo comunidades nativas y unidades de conservación naturales; motivo por el cual no fue aprobado hasta ahora por el Ministerio de Transporte y Comunicaciones del Perú. En Brasil, los impactos ambientales son esperados, más allá del propio corredor ferroviario. Conforme relatado en la literatura, el proceso de decisión para los corredores de transporte es complejo y exige estudios de viabilidad económica, técnica y ambiental. Las políticas públicas, sociales y ambientales incluidas en el plan de transporte exigen un gran volumen de datos, actores y opiniones que casi siempre contradictorias, que actualmente requieren herramientas de geoprocesamiento para permitir resultados de calidad y en tiempo hábil. No obstante, no hay protocolos oficiales de SIG para ser utilizados por las autoridades de transporte y comunidades locales con respecto a los escenarios predictivos de los impactos ambientales de la ferrovía bioceánica Perú - Brasil. En este contexto, el artículo propone y analiza un modelo para calcular corredores ambientalmente viables considerados en el planeamiento de la ferrovía bioceánica. La solución reúne técnicas AHP de análisis multicriterio a una estructura GIS. Los resultados permiten una fácil visualización comparativa de las alternativas y calcula el camino mejor viable. La organización de los datos y la transparencia en las reglas de decisión son dos atracciones principales del modelo. La solución es flexible, puesto que permite que las reglas sean moldeadas dinámicamente, lo cual posibilita la reproducción del estudio.

Palavras-chave: Corredores de Viabilidad; Planeamiento de Transporte; Geoprocesamiento; Dinamica EGO.

\section{Uma perspectiva ambiental no planejamento da ferrovia bioceânica: modelagem quantitativa de cenários}

Em outubro de 2015, a China Railway Eryuan Engineering Group Co. LTD, realizou um estudo técnico para a construção da ferrovia bioceânica Peru-Brasil. O estudo propôs a rota norte como a melhor alternativa viável. No entanto, essa alternativa foi criticada, pois o projeto colocou em risco comunidades nativas e unidades de conservação natural; motivo pelo qual ainda não foi aprovado pelo Ministério dos Transportes e Comunicações do Peru. No Brasil, são esperados impactos ambientais, além do próprio corredor ferroviário. Conforme relatado na literatura, o processo de decisão para os corredores de transporte é complexo e reque estudos de viabilidade econômica, técnica e ambiental. As políticas públicas, sociais e ambientais incluídas no plano de transporte exigem um grande volume de dados, atores e opiniões quase sempre contraditórias, que atualmente exigem ferramentas de geoprocessamento para permitir resultados de qualidade e em tempo hábil. No entanto, não há protocolos oficiais de SIG a serem usados ??pelas autoridades de transporte e comunidades locais com relação aos cenários preditivos dos impactos ambientais da ferrovia bioceânica Peru-Brasil. Nesse contexto, o artigo propõe e analisa um modelo para calcular corredores ambientalmente viáveis, considerados no planejamento da ferrovia bioceânica. A solução reúne técnicas de análise multicritério AHP em uma estrutura GIS. Os resultados permitem uma fácil visualização comparativa das alternativas e calculam o melhor caminho viável. A organização dos dados e a transparência nas regras de decisão são duas atrações principais do modelo. A solução é flexível, pois permite que as regras sejam moldadas dinamicamente, o que possibilita a reprodução do estudo.

Palavras-chave: Corredores de viabilidade; Planejamento de Transporte; Geoprocessamento; Dinâmica do EGO.

Topic: Tecnologia, Modelagem e Geoprocessamento

Reviewed anonymously in the process of blind peer.

\section{Miluska Blas Leon (iD}

Universidade Federal de Minas Gerais, Brasil

http://lattes.cnpq.br/2567004794270537

http://orcid.org/0000-0002-1440-9638

miluskablasbr@gmail.com

Rodrigo Affonso Alburquerque Nóbrega (iD)

Universidade Federal de Minas Gerais, Brasil

http://lattes.cnpq.br/7158751194696023

http://orcid.org/0000-0001-7058-5903

raanobrega@ufmg.com.br

William Leles de Sousa Costa (iD)

Universidade Federal de Minas Gerais, Brasil

http://lattes.cnpq.br/5023169180168026

http://orcid.org/0000-0002-6117-4819

williamleles@gmail.com
Received: $17 / 03 / 2020$

Approved: 22/04/2020
Referencing this:

LEON, M. B.; NÓBREGA, R. A. A.; COSTA, W. L. S.. Una perspectiva ambiental en el planeamiento de la ferrovía bioceánica: modelamiento cuantitativo de escenarios. Revista Ibero Americana de Ciências Ambientais, v.11, n.3, p.454-468, 2020. DOI: http://doi.org/10.6008/CBPC2179-6858.2020.003.0035 


\section{INTRODUCCIÓN}

En el año 2008 , se crea la ley $\mathrm{N}^{\circ} 29207$, que declara de necesidad pública y de interés nacional la construcción de la ferrovía transcontinental "Brasil-Perú" atlántico-pacífico (FETAB) con la finalidad de entrelazar los dos océanos de la región Sudamericana; China muestra interés por el proyecto que en mayo de 2015 suscribiendo un memorándum sobre la interconexión ferroviaria bioceánica China - Perú - Brasil (NOVAK et al., 2016). Sin embargo, de acuerdo con Caillaux et al. (2016) uno de los mayores retos es sin duda alguna establecer la viabilidad de dicho proyecto. Según Dorador (2016) menciona que se realizaron estudios tanto para el Brasil como para el Perú llegando a la conclusión, por el lado brasileño existe mayor convicción del trazado de la vía mientras, que por el lado peruano se proyectan una serie de posibilidades con mayor o menor grado de viabilidad. En ambos países, el proyecto de la ferrovía tiene pros y contras. La perspectiva mercadológica y macrologística son los principales impulsores del proyecto, al paso que las perspectivas ambiental y social son las principales limitaciones.

En el informe por la consultora el Perú cuenta con tres posibles rutas (norte, centro y sur) siendo la ruta norte la alternativa viable, desde el punto de vista de la variable ingeniería, esta alternativa definida deberá ser: conforme a las características topográficas a lo largo de la vía para satisfacer las necesidades de transporte y acceso a la construcción, contar con las mejores condiciones geológicas y menores costo de inversión (CREEG, 2015). Sin embargo, esta alternativa recibió numerosas críticas tanto a nivel nacional como internacional por sus presuntos efectos nefastos al Parque Nacional Cordillera del Divisor y a la Reserva Territorial Isconagua; por ello, la Oficina de Programación de Inversiones del Ministerio de Transporte y Comunicaciones del Perú, determinó que el estudio del perfil del proyecto no cuenta con los elementos y el sustento necesarios para su aprobación (NOVAK et al., 2016)

Según Arce et al. (2010), los estudios en proyectos de transporte deben tener en cuenta los aspectos sociales, físicos y económicos junto a los ambientales, de manera que el equilibrio entre esos cuatro factores permita avanzar hacia la sostenibilidad. Se supone que, si la evaluación de cada etapa no es favorable o si en ella no se subsanaron las deficiencias y observaciones de la etapa anterior, el proyecto no puede seguir adelante. En general, el componente físico es razonablemente bien hecho, pero los otros pueden no existir o son manipulados los datos para concluir que la obra sea viable. Según Dourojeanni et al. (2009) el proceso de decisiones no siempre responden a ofertas ni a demandas reales, son captadas por sectores empresariales, que para subsistir, apenas necesitan "hacer" no importa "qué".

De acuerdo con Carrasco et al. (2010), en la planificación de la evaluación de impacto ambiental eran incorporado estas variables ambientales en fases muy tardías, cuando las actuaciones, los "proyectos" podrían estar ya demasiado acotados o definidos. Según Quintero (2009) menciona que desde la planificación hasta la operación, se requiere una evaluación de los impactos ambientales que podrían causar al medio ambiente. Idealmente, los proyectos deben evitar, a continuación, minimizar, luego, restaurar y finalmente cuando se hayan agotado las opciones anteriores compensar, esto se conoce como Jerarquía de mitigación (BAGER, 2015). Es por ello para evitar el impacto en áreas sensibles, lo mejor es excluirlas, está debe ser la 
opción preferida que debe tomarse para evitar la creación de impactos desde un principio (SADASIVUNI et al., 2009).

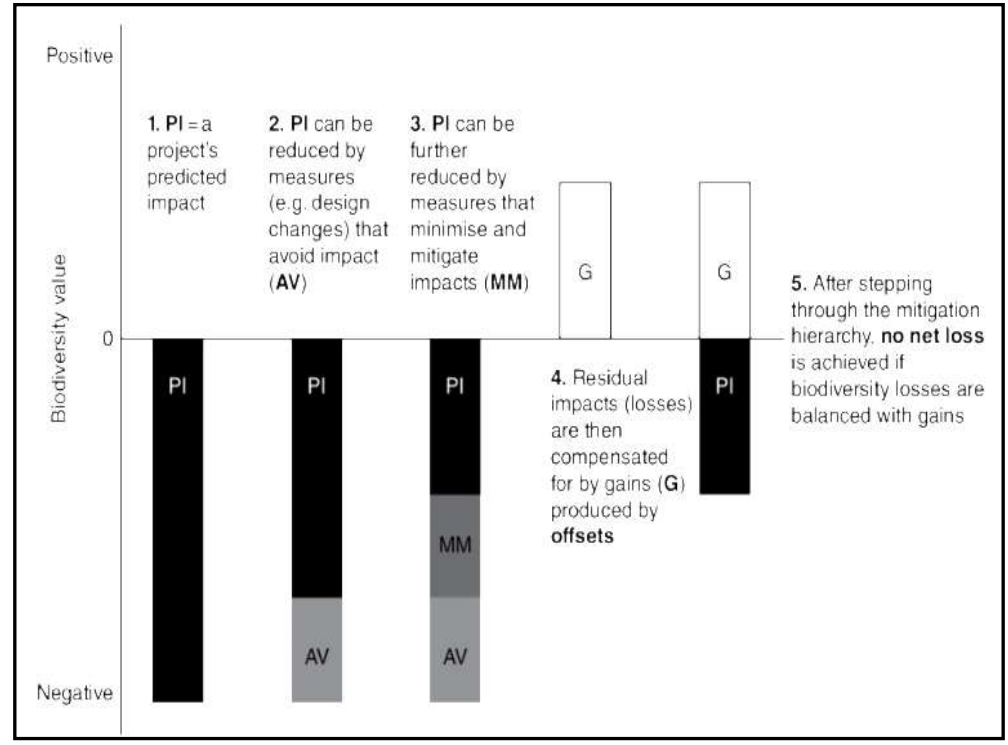

Figura 1: Los pasos secuenciales de la aplicación de la jerarquía de mitigación (de izquierda a derecha) y el papel de las compensaciones para garantizar 'sin pérdida neta'. Fuente: Adaptado por BBOP (2009) y Quétier et al. (2012)

El proceso de toma de decisiones en el planeamiento de transporte envuelve actualmente una gama mayor de variables, de actores y de regulaciones comparando a forma tradicional practicada en el pasado (NÓBREGA et al., 2011) que según esa complejidad de las políticas de transporte es el resultado de las políticas ambientales y sociales incluyendo la participación pública en el proceso.

En la toma de decisiones todos los aspectos implicados en la planificación del transporte no deben ser evaluados aisladamente, es por ello que los sistemas de información geográfica juegan un papel especial por la capacidad de almacenar y procesar bases de datos de grande soporte y permitir modelamiento de escenarios complejos para el análisis y toma de decisión (SADASIVUNI et al., 2009; NÓBREGA, 2016). En análisis ambientales, las herramientas de geoprocesamiento permiten realizar mediciones y cálculos con una mayor precisión y objetividad que si se realizan sin su ayuda (ARCE et al., 2010).

Sin embargo, la aplicación de métodos de evaluación multicriterio en la fase de planificación de grandes infraestructuras de transporte resulta habitual el uso de mapas temáticos que representan aspectos del territorio que se consideran sensibles y la "superposición" de los diferentes corredores de transporte con los mapas temáticos permite, por un lado, evitar las zonas a proteger y, por otro, facilitar la evaluación de la magnitud que tendría el impacto provocado por las infraestructuras sobre el territorio (ARCE et al., 2010)

En ese contexto el presente artículo tiene como objetivo general proponer y analizar un modelo del corredor de viabilidad ambiental en el planeamiento de la ferrovía Bioceánica Perú - Brasil y como objetivos específicos: Modelar corredores de viabilidad ambiental apoyados en geoprocesamiento y análisis de multicriterio como ruptura de paradigma en el proceso de planeamiento ferroviario; Construir y comparar métricas de dos escenarios con prioridad ambiental y no prioridad ambiental para garantizar el nivel de prevención de la jerarquía de mitigación. 


\section{MATERIALES Y MÉTODOS}

\section{Localización del área}

La presente investigación utiliza la misma área de estudio presentado por la consultora (CREEG, 2015) y tiene como punto de partida el municipio de Rio Branco en el estado de Acre, punto viable de convergencia para la ruta oeste, a partir del cual fueron desarrollados diferentes ensayos hasta alcanzar el océano pacífico. En el Perú se propuso diferentes puntos de llegada, no se trata como punto fijo a un puerto marítimo de destino, sino que se considera tres opciones de puertos peruanos: puerto del Callao, puerto de llo y el puerto de Matarani. De esa forma el modelo puede tener libertad de calcular los corredores sobre la base de criterios de mínimo esfuerzo o costo, para posterior comparación de los resultados sobre una misma base de datos. La figura 2 ilustra el área de estudio.

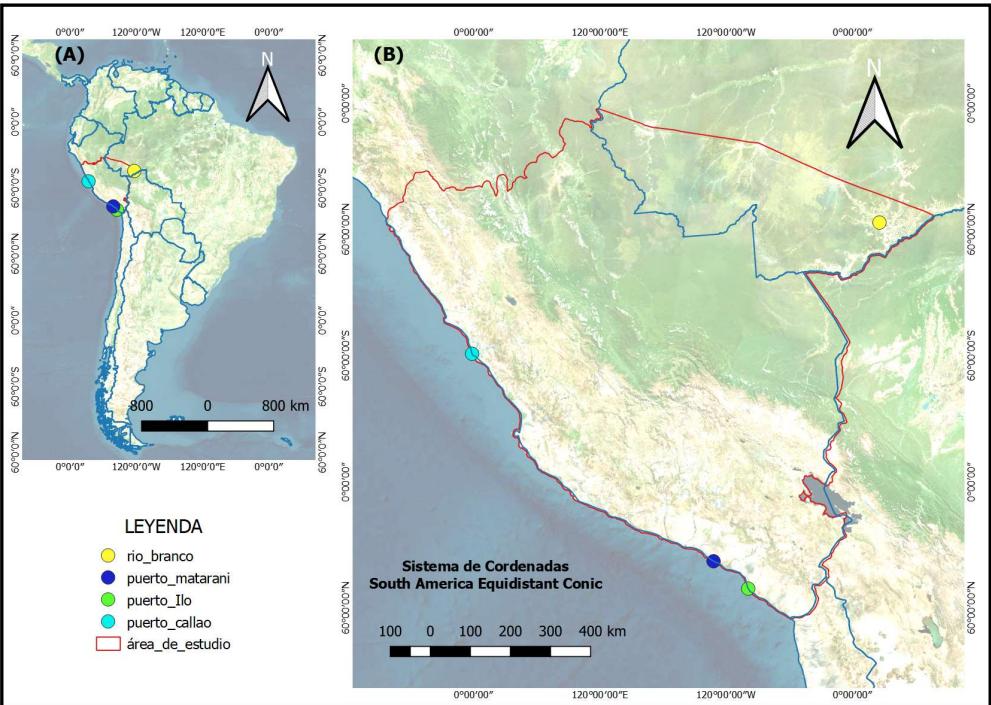

Figura 2: (A) Localización del área de estudio en relación al mapa sudamericano. (B) Localización del área de estudio centro- sur del Perú y el Estado de Acre en Brasil

El área de estudio tiene una gran complejidad en ambos países, sin embargo, la heterogeneidad de los contextos social, económico, cultura y principalmente físico en territorio peruano eleva el grado de dificultad y de complejidad del proyecto. El área forestal se encuentra representada por variedad de formas de vida vegetal o formas de crecimiento, distribuidas en zonas de vida, a partir del mapa de cobertura vegetal del Ministerio de Ambiente del Perú 2015 y Map Biomas del Brasil el área de estudio fue clasificada en: bosques costeros que representa el $0,69 \%$, bosques de selva baja que representa $23 \%$ y selva alta $8 \%$ y bosques andinos el $0,01 \%$ y los bosques amazónicos en Acre que representa el 16\%.

En la región de estudio se encuentran las Áreas Naturales protegidas tanto para el Perú como para el Brasil. SERNAP (2018) menciona, el Perú cuenta con 75 Áreas Naturales Protegidas informe hasta el año 2017 de las cuales 37 se encuentran en el área de estudio y el Brasil - Acre según, WWF Brasil (2009) son 19 en el área de estudio, denominadas, Unidades de Conservación.

El Perú y el Brasil cuenta con comunidades nativas según el censo INEI (2018) el Perú cuenta con 2703 comunidades, ocupando los ámbitos de Selva y Ceja de Selva de 11 departamentos del territorio peruano, las cuales siete departamentos se encuentran en el área de estudio: Ayacucho, Cusco, Huánuco, Junín, Madre 
de Dios, Pasco, y Ucayali. Los departamentos que presentan mayor número de comunidades nativas de acuerdo al área de estudio es Junín (285), Pasco (183) y Cusco (100); mientras que Ayacucho (10) es el departamento que registra el menor número de comunidades nativas, que representa el $0,4 \%$. Según PROACRE (2012) Acre posee 36 tierras indígenas (TIs) distribuidas en 11 de los 22 municipios acreanos. En ellas viven 15 pueblos indígenas además cuatro diferentes grupos considerados "aislados".

Otro aspecto importante y muy complejo es la topografía y morfología del terreno que varía desde la planicie amazónica hasta la costa peruana traspasando obligatoriamente la Cordillera de los Andes. En la región peruana está localizada la cordillera Tíclio que es un paso montañoso de los Andes peruanos que atraviesa la ruta nacional PE-22, también conocida como la Carretera Central en el Km 120 alcanza su punto de mayor altitud 4818 m.s.n.m. Sin embargo, no es apenas la altitud y la inclinación del terreno que aumentan el grado de dificultad del proyecto, puesto que la porción amazónica del área de estudio presenta extensas áreas de suelos arcillosos, resultantes de la combinación de factores pedológicos e hidrológicos ya mencionados durante la construcción de la Estrada de Ferro Madeira Mamoré (CRAIG, 1947). Además, las regiones serranas del Brasil, existe una preocupación elevada con el uso y ocupación del suelo, una vez que son áreas en que el proceso erosivo se da de forma acelerada (BARBOSA et al., 2019), que es una desventaja para el corredor ferroviario.

Todavía la idea de una conexión logística de alta capacidad de carga interconectado la red ferroviaria brasilera a un puerto en el pacífico en la costa peruana ha sido perseguido durante mucho tiempo y es el principal motivador de esta investigación. La premisa es que la ruta bioceánica opera como un canal para el transporte de carga, históricamente productos minerales y agrícolas hacia el pacífico y la entrada de productos extranjeros, especialmente desde Asia. También existe la premisa de que la infraestructura ferroviaria fortalece el comercio exterior entre los dos países.

En el caso de factores económicos el Perú es el primer productor latinoamericano de zinc y plomo; y el segundo productor a nivel mundial de zinc, cobre y plata; el año 2018 el nivel de exportación aumento a 0,1\% en plata además de productos no metálicos a 1,3\% en comparación al año 2107 (MINEM, 2018). Según MINAGRI (2018) menciona, la producción agrícola en el Perú creció en 9,9\% comparado al año 2017 y 2018, algunos productos de exportación son el maíz amarillo duro que aumento en 17\% (en Ancash, San Martín e Ica), el café pergamino en 9\% (en Junín, San Martín y Pasco), la uva 9\% (en Ica y Lambayeque). El factor económico y minero es fundamental ya que genera una atracción favorable para el corredor de la ferroviaria Bioceánica Perú - Brasil.

En el Brasil, la región centro - oeste es la principal productora de granos como soya y maíz y grande parte de esa producción es destinada a mercados internacionales, incluyendo a China. Cabe señalar que la producción agrícola en esta región ha batido records año tras año, no solo por la expansión del área de plantaciones, sino también por el aumento de la productividad (HOPEWELL, 2017). La salida de estos productos se ha llevado a cabo tradicionalmente hecho por carreteras que cubren distancias de $1800 \mathrm{~km}$ en promedio a los puertos del Océano Atlántico (HERNÁNDEZ, 2011). La construcción de ferrocarriles que conectan la región con la red ferroviaria existente que converge en el puerto de Santos está en marcha, y un 
proyecto ferroviario hacia el puerto fluvial de Miritituba en el estado de Pará (NÓBREGA et al., 2016). Tales empresas demuestran la viabilidad económica de una solución marco para la región, incluso sin considerar los productos mineros. Los factores positivos, como el desarrollo económico y macrologístico, tienen un peso significativo en el proceso de toma de decisiones y casi siempre son la demanda de estudios de viabilidad para proyectos de infraestructura. Sin embargo, la planificación contemporánea del transporte no puede limitarse a la perspectiva de ingeniería tradicional (NÓBREGA et al., 2011; NÓBREGA et al., 2016). Los aspectos sociales, culturales y ambientales son de vital importancia para la conservación, prevención, orientación y repulsión de obra de infraestructura, optimizando el proyecto para servir mejor a la sociedad de forma sustentable. El grande desafío es reunir diferentes actores y sus opiniones y objetivos normalmente divergentes por el bien de la definición de un corredor ferroviario eficiente y con un trazado de mínimo costo ambiental.

El estudio tiene un enfoque ambiental y como tal fueron seleccionadas ocho variables (pendiente, comunidad nativa, concesionarias mineras, agricultura, carreteras, arqueología, usos de conservación integral y uso de conservación sostenible), para la creación de los escenarios. Todas las variables se obtuvieron a través de datos geográficos de fuentes públicas y de libre acceso, algunas en formato vector y otras imágenes. Todos los datos fueron convertidos al formato matricial en ArcGis 10.4.1, después para el modelamiento geográfico se realizó en un software libre DINAMICA EGO (Environment for Geoprocessing Objects), que consiste en una plataforma de modelamiento que es ejecutada por medio de operadores matemáticos llamados "functores", modelos espacialmente explícitos, además de utilizar algoritmos para la calibración y validación de las simulaciones (SOARES FILHO et al., 2009). se usó Excel para el análisis multicriterio y finalmente se representó los corredores ferroviarios en QGIS 3.2.0.

La metodología del estudio fue adaptada del modelo propuesto por Nóbrega et al. (2016). El estudio está dividido en cuatro etapas, iniciando por la obtención y tratamiento de los datos geográficos, el procesamiento o adecuación de los datos para la entrada en el modelo, el modelamiento de los datos geográficos, el análisis multicriterio y cálculo de los escenarios alternativos de corredores para la viabilidad ambiental de la Ferrovía Bioceánica. A continuación, se presenta un esquema:

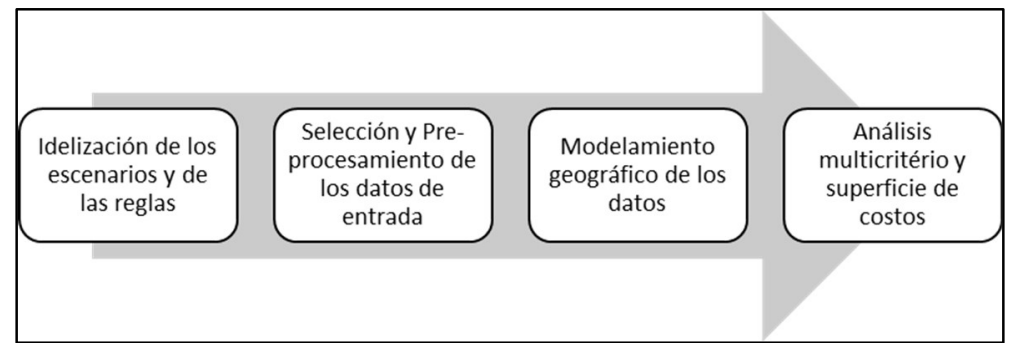

Figura 3: Flujo de etapas del modelo de inteligencia geográfica para procesamiento de corredores de transporte.

Fuente: Nóbrega et al. (2016).

Idealización de los escenarios y de las reglas para el modelamiento

Esta etapa corresponde al modelamiento conceptual, y es la etapa responsable por el diseño metodológico a ser implementado en el modelamiento lógico. En este estudio fueron idealizados tres 
escenarios conectando los puertos del Callao, Ilo y Matarani en el Perú con Rio Branco en Brasil. El primer escenario fue representado con pesos altos para los factores ambientales, el segundo escenario representado con pesos altos para los factores no ambientales y el tercer escenario con pesos equitativos.

Tabla 1: Técnicas de geoprocesamiento para la preparación de los datos de entrada.

\begin{tabular}{|l|l|}
\hline \multicolumn{1}{|c|}{ Herramientas } & \multicolumn{1}{c|}{ Descripción } \\
\hline $\begin{array}{l}\text { Projections and Transformations/ } \\
\text { Proyección y Transformación }\end{array}$ & $\begin{array}{l}\text { Proyectar datos espaciales de un sistema de coordenadas a otro. El sistema de } \\
\text { coordenada que se uso es South_America_Equidistant_Conic }\end{array}$ \\
\hline $\begin{array}{l}\text { Mosaic to New Raster/ Mosaico de } \\
\text { Nuevo Ráster }\end{array}$ & Funciona múltiples datsets ráster en un nuevo ráster. \\
\hline $\begin{array}{l}\text { Distance Euclidean/Distancia } \\
\text { Euclidiana }\end{array}$ & $\begin{array}{l}\text { Para el análisis espacial se requieren reglas de proximidad generando una matriz } \\
\text { donde las distancias se representan continuamente. }\end{array}$ \\
\hline Clip/Recorte & $\begin{array}{l}\text { Se usa para recortar los diversos datos geográficos de entrada de acuerdo con los } \\
\text { límites establecidos por el área de estudio. }\end{array}$ \\
\hline Slope/Pendiente & Identifica la pendiente de cada celda de una superficie. \\
\hline Reclass/Reclasificación & $\begin{array}{l}\text { Reclasificación de datos continuos para comprender las clases como categorías en } \\
\text { mapas temáticos. }\end{array}$ \\
\hline $\begin{array}{l}\text { Conversion to raster Conversión a } \\
\text { Ráster }\end{array}$ & Convertir de un shapefile (punto, polígono y polilínea) en ráster. \\
\hline
\end{tabular}

En la etapa de pre-procesamiento, los datos seleccionados son preparados/ modificados para la entrada del modelo. El primer paso fue el recorte geográfico de los datos usando el polígono del área de estudio, otros datos fueron necesario hacer mosaico para reunir dicha área, como es el caso del modelo digital de elevación y finalmente homogenizar todos los datos en un mismo sistema de coordenadas (South_America_Equidistant_Conic) con un pixel 1000 metros para luego generar mapas temáticos y ser convertido en formato matricial. La resolución espacial de 1000 metros permite que el modelo sea sensible a todas las características ambientales y fenómenos compatibles con la escala y alcance del proyecto, sin comprometer significativamente el desempeño computacional del modelo.

\section{Modelamiento Geográfico}

El procedimiento del modelo geográfico, comprende el tratamiento de cada mapa temático de entrada para que luego puedan ser procesados y analizados en conjunto. Sin embargo, el desafío inicial es entender el problema y como estos mapas deberán ser analizados para proponer soluciones; es por ello que se establece reglas para modelar cada mapa temático y establecer parámetros. Los datos geográficos posibilitan un análisis por cada variable que lo integra, estas variables son trabajadas en un proceso jerárquico responsable por la atribución de pesos de acuerdo con las características distintitas de cada una. La ponderación de las variables ocurre en tres aspectos: i. Inicialmente los pesos son utilizados para modelar los datos brutos de entrada y convertirlos en datos matriciales en el modelo; ii. Las variables, agrupadas por conjunto, son ponderadas y combinadas entre sí para generar una superficie integrada de esfuerzo por conjunto; iii. Una vez definida las superficies integradas de esfuerzo por conjunto de variables estas son combinadas utilizando ponderaciones ofrecidas por gestores y demás interesados en el proyecto. Diferentes combinaciones de pesos generan diferentes escenarios.

Para mayor información del procedimiento se encuentra en, (SADASIVUNI et al., 2009; NÓBREGA et al., 2016; NÓBREGA et al., 2017) y ver los cuadros 1, 2, 3. 


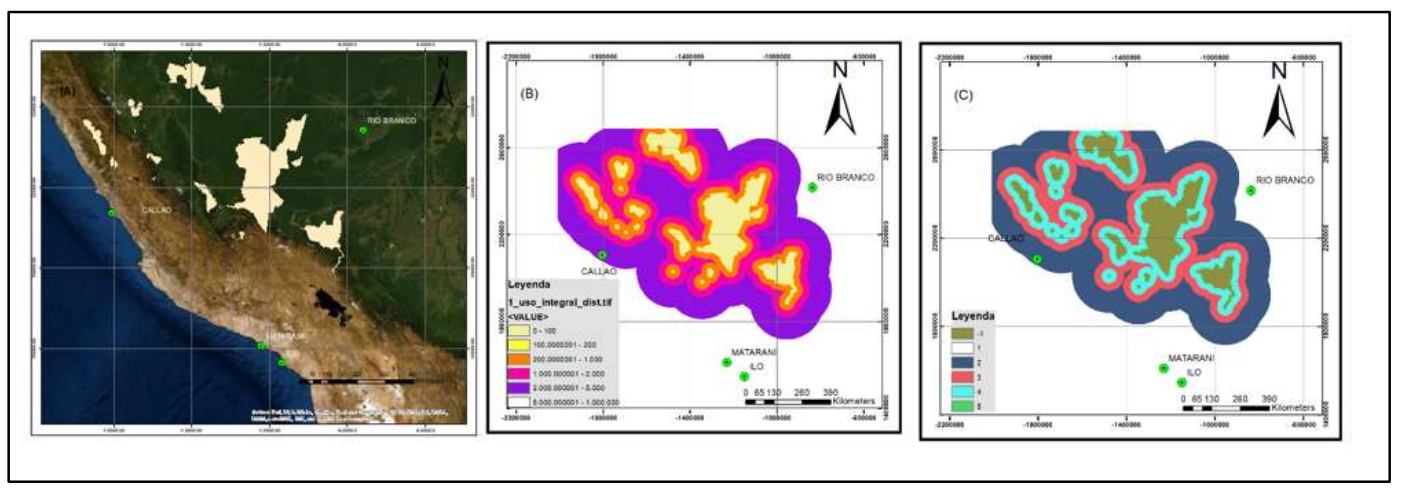

Figura 4: Ejemplo de modelamiento geográfico para conectar la ferrovía bioceánica entre Perú y Brasil: (A) Mapa de Protección de Uso integral (B) Mapa Euclidiana de Protección Uso Integral (C) Mapa de ponderación AHP.

Cuadro 1: Variables ambientales utilizados para el modelamiento geográfico de los corredores ferroviarios y sus respectivas reglas

\begin{tabular}{|c|c|c|c|c|c|}
\hline Factores & Variable & Regla & $\begin{array}{c}\text { Modelamiento } \\
\text { Conceptual }\end{array}$ & Modelamiento Lógico / Computacional & $\begin{array}{c}\text { Parámetro } \\
\text { AHP }\end{array}$ \\
\hline AMBIENTAL & U.C Integral & Repulsión & $\begin{array}{l}\text { Impedir la ocupación } \\
\text { de áreas de la U.C } \\
\text { Integral. }\end{array}$ & $\begin{array}{l}\text { Mapa de distancia Euclidiana. Las distancias menores } \\
\text { positivos son más atractivas al emprendimiento del } \\
\text { corredor ferroviario, recibiendo así factores AHP } \\
\text { menores; por } \\
\text { contrario, a mayor distancia son menos atractivas por } \\
\text { ende reciben factores de AHP mayores. }\end{array}$ & $\begin{array}{l}0,-1 \\
1,5 \\
501,4 \\
1001,3 \\
2001,2 \\
3001,1\end{array}$ \\
\hline & $\begin{array}{l}\text { U.C } \\
\text { Sustentable }\end{array}$ & Repulsión & $\begin{array}{l}\text { Impedir la ocupación } \\
\text { de áreas de la U.C } \\
\text { Sustentable. }\end{array}$ & $\begin{array}{l}\text { Mapa de distancia Euclidiana. Las distancias menores } \\
\text { positivos son más atractivas al emprendimiento del } \\
\text { corredor ferroviario, recibiendo así factores AHP } \\
\text { menores; por } \\
\text { contrario, a mayor distancia son menos atractivas por } \\
\text { ende reciben factores de AHP mayores. }\end{array}$ & $\begin{array}{l}\text { AHP: } \\
0,-1 \\
1,5 \\
501,4 \\
1001,3 \\
2001,2 \\
3001,1\end{array}$ \\
\hline
\end{tabular}

Cuadro 2: Variables económicos y logísticos utilizados para el modelamiento geográfico de los corredores ferroviarios y sus respectivas reglas

\begin{tabular}{|c|c|c|c|c|c|}
\hline Factores & Variable & Regla & $\begin{array}{c}\text { Modelamiento } \\
\text { Conceptual }\end{array}$ & Modelamiento Lógico / Computacional & $\begin{array}{c}\text { Parámetro } \\
\text { (AHP) }\end{array}$ \\
\hline \multirow[t]{3}{*}{$\begin{array}{l}\text { ECONÓMICO / } \\
\text { LOGISTICO }\end{array}$} & Minería & Atracción & $\begin{array}{l}\text { Es una variable } \\
\text { atractiva para la } \\
\text { ferrovía. }\end{array}$ & $\begin{array}{l}\text { Mapa de distancia Euclidiana. Las distancias son } \\
\text { divididas en } 4 \text { clases para la atribución de los factores } \\
\text { AHP. Distancias menores son más atractivas para el } \\
\text { emprendimiento del corredor ferroviario. Recibirán } \\
\text { factores AHP menores. }\end{array}$ & $\begin{array}{l}0,1 \\
61,2 \\
91,4 \\
121,6\end{array}$ \\
\hline & Carretera & Atracción & $\begin{array}{l}\text { Es una variable } \\
\text { atractiva para la } \\
\text { ferrovía. }\end{array}$ & $\begin{array}{l}\text { Mapa de distancia Euclidiana. Las distancias son } \\
\text { divididas en } 4 \text { clases para la atribución de los factores } \\
\text { AHP. Distancias menores son más atractivas para el } \\
\text { emprendimiento del corredor ferroviario. Recibirán } \\
\text { factores AHP menores. }\end{array}$ & $\begin{array}{l}0,1 \\
61,2 \\
91,4 \\
121,6\end{array}$ \\
\hline & Agricultura & Atracción & $\begin{array}{l}\text { Es una variable } \\
\text { atractiva para la } \\
\text { ferrovía. }\end{array}$ & $\begin{array}{l}\text { Mapa de distancia Euclidiana. Las distancias son } \\
\text { divididas en } 4 \text { clases para la atribución de los factores } \\
\text { AHP. Pesos menores son más atractivas para el } \\
\text { emprendimiento del corredor ferroviario. }\end{array}$ & $\begin{array}{l}0,1 \\
61,2 \\
91,4 \\
121,6\end{array}$ \\
\hline
\end{tabular}

Cuadro 3: Variables físico y cultural utilizados para el modelamiento geográfico de los corredores ferroviarios y sus respectivas reglas

\begin{tabular}{|c|c|c|c|c|c|}
\hline Factores & Variable & Regla & $\begin{array}{l}\text { Modelamiento } \\
\text { Conceptual }\end{array}$ & Modelamiento Lógico / Computacional & $\begin{array}{c}\text { Parámetro } \\
\text { (AHP) }\end{array}$ \\
\hline FISICO & $\begin{array}{l}\text { Modelo Digital } \\
\text { de terreno }\end{array}$ & Repulsión & $\begin{array}{l}\text { Impedir el paso de la } \\
\text { ferrovía por mayor } \\
\text { pendiente }\end{array}$ & $\begin{array}{l}\text { Mapa de pendiente en porcentaje. La pendiente } \\
\text { será presentada en } 7 \text { clases. La atribución de los } \\
\text { factores será directamente proporcional al } \\
\text { aumento del valor de la pendiente del terreno. }\end{array}$ & $\begin{array}{l}0,1 \\
5,1 \\
10,2 \\
15,3 \\
30,4 \\
50,5 \\
1000,10\end{array}$ \\
\hline \multirow[t]{2}{*}{ CULTURAL } & Arqueología & Repulsión & $\begin{array}{l}\text { Impedir la ocupación de } \\
\text { áreas arqueológica. }\end{array}$ & $\begin{array}{l}\text { Mascara restrictiva sobre el polígono. Mapa de } \\
\text { distancia. AHP mayor } 5 \text {. Distancias mayores son } \\
\text { menos atractivas para la variable arqueología. }\end{array}$ & $\begin{array}{l}0,-1 \\
250,3 \\
500,5\end{array}$ \\
\hline & Arqueología & Repulsión & $\begin{array}{l}\text { Impedir la ocupación de } \\
\text { áreas arqueológica. }\end{array}$ & $\begin{array}{l}\text { Mascara restrictiva sobre el polígono. Mapa de } \\
\text { distancia. AHP mayor } 5 \text {. Distancias mayores son } \\
\text { menos atractivas para la variable arqueología. }\end{array}$ & $\begin{array}{l}0,-1 \\
250,3 \\
500,5\end{array}$ \\
\hline
\end{tabular}




\section{Análisis Multicriterio}

Es un método de comparación por pares para la toma de decisiones de múltiples criterios, este método emplea el análisis de decisiones matemáticas para determinar las prioridades de varias alternativas mediante la comparación por pares de diferentes elementos de decisión con referencia a un criterio común. Para la solución al problema se dividió en tres categorías: objetivo, factores y criterios.

En síntesis, el objetivo del análisis multicriterio es determinar corredores de viabilidad ambiental en el proceso de planeamiento de la ferroviaria Perú- Brasil. Los factores corresponden a una forma con que los análisis serán desarrollados, incluyendo las variables y las reglas de decisión para cada variable (SADASIVUNI et al., 2009). Debido a la complejidad y de la diversidad de temas envueltas en estudios de viabilidad de ferrovías, Nóbrega et al. (2016) sugiere la clasificación de los factores en diferentes grupos de variables para proteger el modelo de ponderaciones con sesgo cuando se utilizan técnicos de diferentes áreas. En ese sentido, este trabajo agrupa los factores en cuatro clases en: Ambiental, Social/Cultural, Físico y Económico/ Logístico y los criterios mencionados anteriormente son clasificado por cada factor. A continuación, se presenta el siguiente esquema:

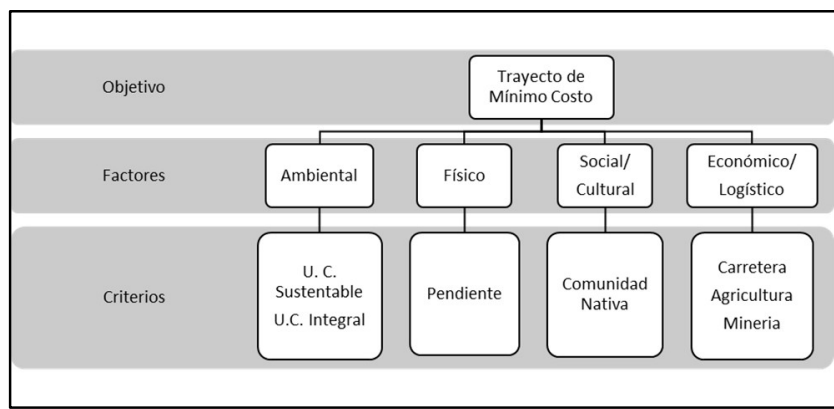

Figura 5: Proceso de Análisis Jerárquico. (AHP). Fuente: Sadasivuni et al. (2009).

Los criterios son ejecutados en un proceso jerárquico de análisis multicriterio (AHP), para ello se le asigna una puntuación entre 1 y 9 para dar una calificación relativa, los valores asignados son tomados por especialistas (SAATY et al., 2012). De acuerdo con Sadasivuni et al. (2009) menciona que la escala de puntuación diferencial supone que el criterio de fila es de igual o mayor importancia que el criterio de columna. Los valores recíprocos $(1 / 3,1 / 5,1 / 7$ o 1/9) se han utilizado cuando el criterio de fila es menos importante que el criterio de columna, en conclusión, los valores menores representaran en el modelo atracción (mayor viabilidad) y valores mayores representaran repulsión (baja viabilidad o imposibilidad).

Como resultado el modelo genera mapas temáticos integrados. Una vez que se crea la matriz de comparaciones pareadas, se calcula lo que se denomina prioridad. Esta indica la importancia que el decisor le ha asignado a cada elemento. Las prioridades se expresan en forma de vectores que se denomina autovalores o autovectores propios. El método de Saaty de vectores propios/pesos relativos se calcula mediante ecuaciones. A continuación, se calcula su consistencia que se refiere al juicio que muestra el tomador de decisiones en el transcurso de la serie de comparaciones pareadas, solo se da en un caso ideal, ya que existe la subjetividad innata del decisor. Esta subjetividad se intenta hacer lo más objetiva posible en el procedimiento de comparación de matrices pareadas. Para determinar la relación de consistencia (Cr) 
implica habilidad y lógica y si la $\mathrm{Cr}<0,10$, entonces la relación se mantiene consistente, de lo contrario, la relación es inconsistente y el cálculo de los números requiere una revisión hasta que se alcancen los criterios de consistencia. Para mayor detalle se encuentra en (VALEC, 2014).

\section{RESULTADOS}

Al integrar las variables geográficas a través del análisis multicriterio (AHP), se obtuvo el trazado de mínimo costo o esfuerzo. La superficie de costo resultante refleja las diferentes prioridades estipuladas por el tomador de decisiones. Para el presente trabajo se clasificó en tres escenarios uno cuando existe como prioridad: variables ambientales y dos como prioridad: variables no ambientales y tres prioridades físicas. Para todos los escenarios, se observa la restricción de los trazados para las Unidades de Conservación y Comunidades Nativas; las más representativas son: el Parque Nacional del Manú, el Parque Nacional Bahuaja Sonene, la Reserva Territorial Madre de Dios y la Reserva Extractivista Chico Mendes. A continuación, se muestra las tres alternativas de partida con respecto al Perú y uno de llegada con respecto al Brasil.

\section{Escenario 1: Prioridad Ambiental}

\section{Alternativa 1: (Puerto Callao - Rio Branco).}

En la figura 6 se observa que dentro de la franja roja se encuentra la Reserva Nor Yauyos Cochas por lo cual el corredor resultante rechaza rotundamente esa franja. El corredor nasce un solo trazado y después es dividido en dos trazados que luego vuelve a unirse hasta conectar con el rio Branco.

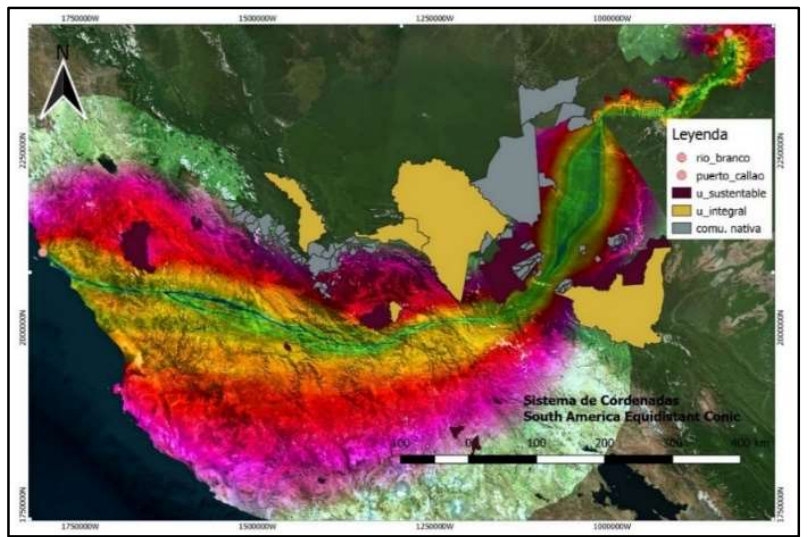

Figura 6: Representación del corredor entre puerto del Callao y rio Branco como prioridad ambiental.

\section{Alternativa 2: (Puerto Matarani - Rio Branco)}

En la figura 7 se observa que repele a las áreas naturales protegidas entre ellas las áreas de Conservación Regional Tres Cañones que se encuentran próximo al corredor. El corredor muestra dos tramos, a los $245 \mathrm{Km}$ y $206 \mathrm{Km}$ los tramos se unen, a partir de los $476 \mathrm{Km}$ de la ruta optima toma la misma ruta que conecta el puerto del callao con Rio Branco como se demuestra en la figura 6, la diferencia está en que el corredor de la figura 7 el trazado es angosto en comparación a la figura 6. 


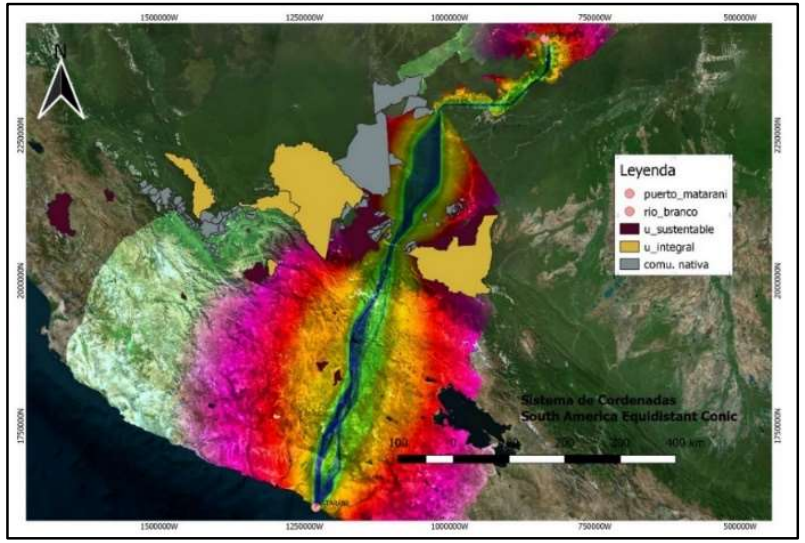

Figura 7: Representación del corredor entre puerto de Matarani y rio Branco como prioridad ambiental.

\section{Alternativa 3: (Puerto llo - Rio Branco)}

En la figura 8 el corredor muestra dos rutas, a partir de los $572 \mathrm{Km}$ de la ruta optima y toma la misma ruta que conecta el puerto del Callao y el puerto de Matarani con Rio Branco.

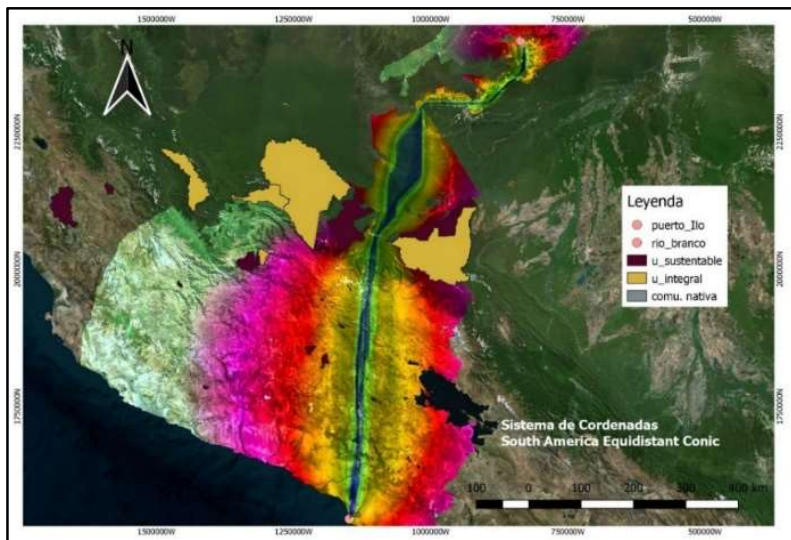

Figura 8: Representación del corredor entre puerto de llo y rio Branco como prioridad ambiental.

\section{Escenario 2: Prioridades No ambientales}

\section{Alternativa 1: (Puerto Callao - Rio Branco).}

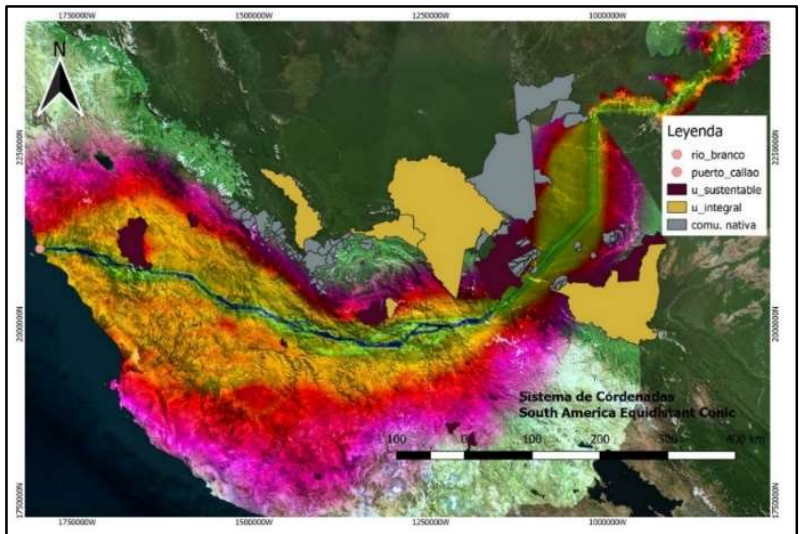

Figura 9: Representación del corredor entre puerto del Callao y rio Branco como prioridad no ambiental.

En la figura 9 se observa que el corredor se encuentra cerca de la Reserva Nor Yauyos Cochas a diferencia de la figura 6 que el corredor está a una distancia aproximada de $23 \mathrm{~km}$ del área natural protegida; este resultado para la figura 8 muestra que el peso usado para las variables ambientales fueron bajos a 
diferencia de la figura 6; además la figura 9 tiene una mayor adherencia o atracción a las zonas mineras y agrícolas, por ende, resulta mayor atracción económica.

\section{Alternativa 2: (Puerto Matarani - Rio Branco)}

En la figura 10 existe una desviación del trazado a comparación de la figura 7, si estas dos se sobreponen; el corredor de la figura 10 se encontraría en una zona que no es prioritario entonces el corredor muestra mayor atracción en zonas donde se encuentran más áreas agrícolas y mineras.

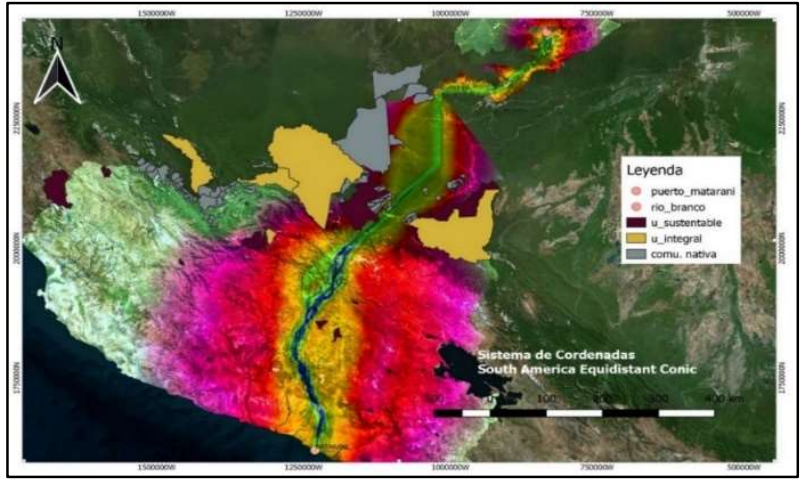

Figura 10: Representación del corredor entre puerto de Matarani y rio Branco como prioridad no ambiental.

\section{Alternativa 3: (Puerto llo - Rio Branco)}

En la figura 11 se observa una desviación del corredor lo cual muestra mayor atracción a las zonas mineras y agrícolas.

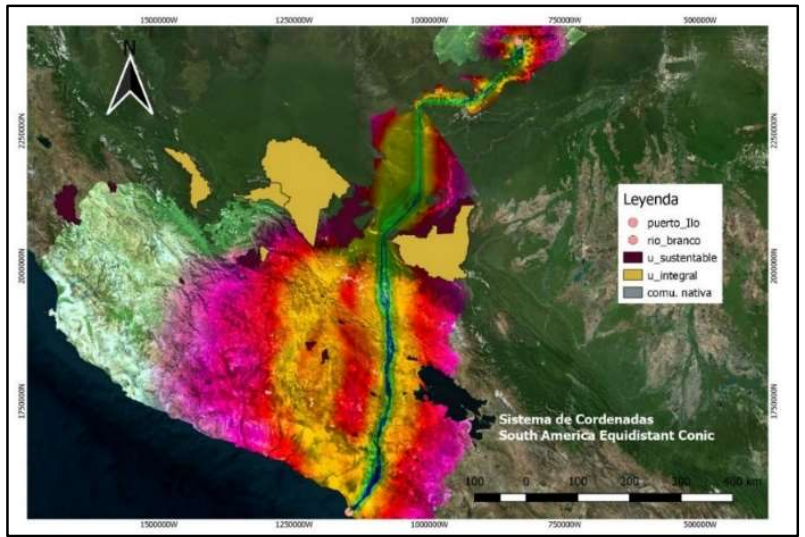

Figura 11: Representación del corredor entre puerto de llo y rio Branco como prioridad no ambiental.

\section{Escenario 3: Prioridad física}

\section{Alternativa 1: (Puerto Callao - Rio Branco).}

Al tomar como prioridad la variable física, se observa en la figura 12 el trazado de la ferrovía comienza por el litoral de la costa peruana por tener una pendiente baja lo cual generaría menos costo de inversión. 


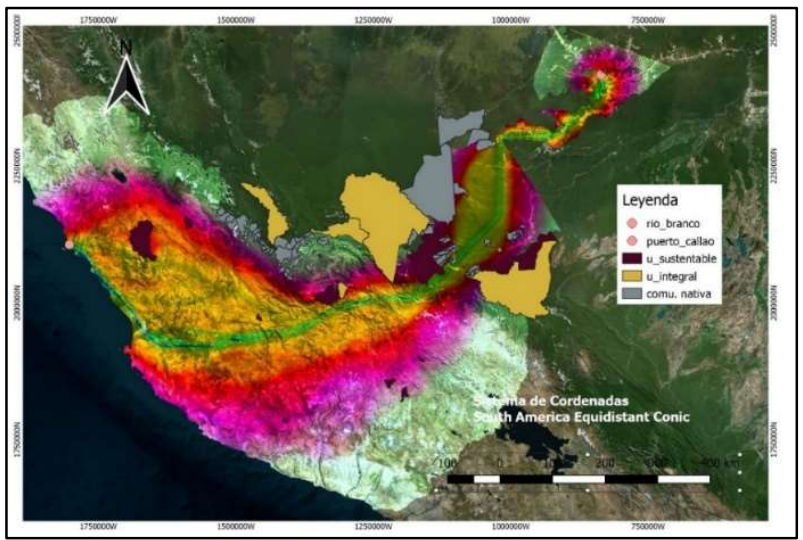

Figura 12: Representación del corredor entre puerto del Callao y rio Branco como prioridad física.

\section{Alternativa 2: (Puerto Matarani - Rio Branco)}

Se muestra en la figura 13, el trazado tiene un desvío leve en comparación con la figura 10 se puede mencionar la figura 13 toma en cuenta la pendiente lo cual generaría menores costo de inversión, mayor aceptación económica por pasar a una cierta distancia por áreas agrícolas y mineras.

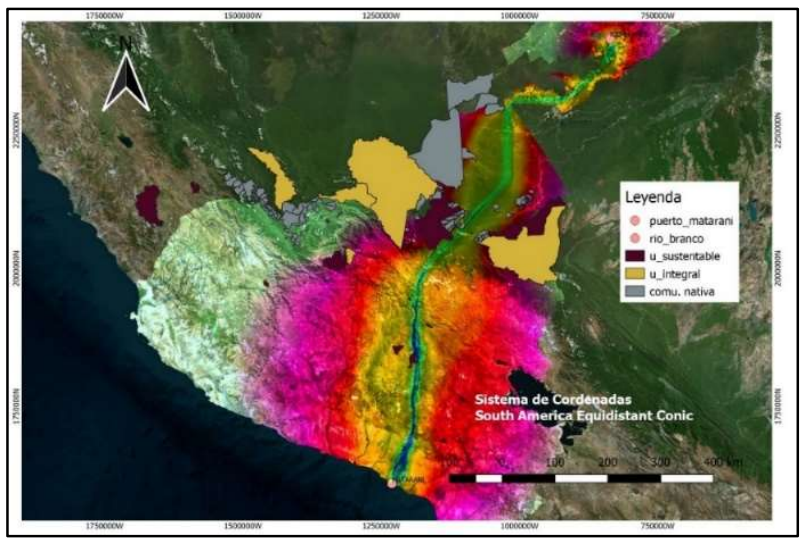

Figura 13: Representación del corredor entre puerto de Matarani y rio Branco como prioridad física.

\section{Alternativa 3: (Puerto llo - Rio Branco)}

La figura 14 muestra tres corredores hasta un cierto tramo que luego en el trayecto se unen a dos y finaliza con un solo corredor. Se observa que uno de los corredores pasa cerca al área natural protegida tres cruces a diferencia de los escenarios figura 8 y 11.

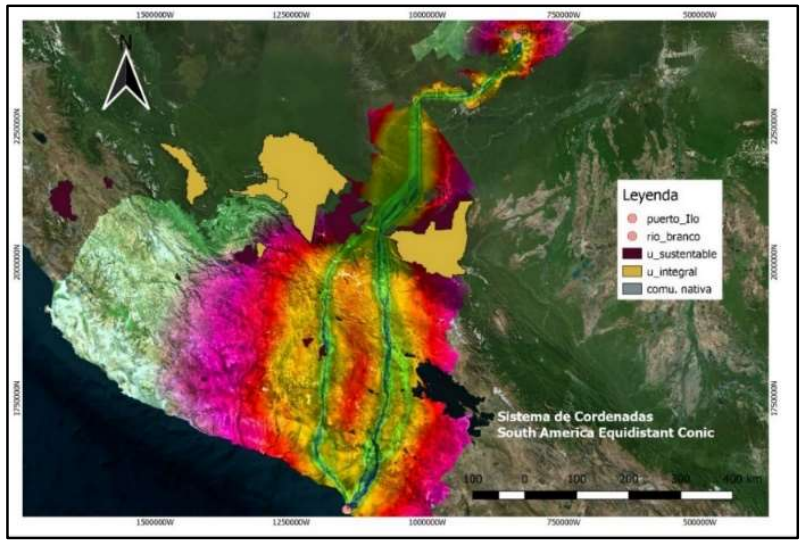

Figura 14: Representación del corredor entre puerto de llo y rio Branco como prioridad no ambiental y pendiente. 


\section{RESULTADOS Y DISCUSIÓN}

Al modelar los corredores de viabilidad ambiental apoyados en geoprocesamiento y análisis multicriterio en el proceso del planeamiento ferroviario se afirma que se obtuvo los posibles corredores, cumpliendo con las reglas establecidas lo cual concuerda con Gonzáles et al. (2012) menciona que el uso de las metodologías de Evaluación Multicriterio, queda comprobada que es una herramienta de alto potencial a la hora de diseñar corredores reales para cualquier tipo de infraestructura lineal y permite minimizar en gran medida el impacto ambiental que pueden llegar a generar. Además según Arce et al. (2010) menciona que en la planificación de grandes infraestructuras de trasporte resulta útil el uso de mapas temáticos que representan aspectos del territorio permitiendo evitar las zonas a proteger.

Al construir y comparar los escenarios con prioridades ambientales y no prioridades ambientales, las superficies de costo tienen implicaciones directas en el trazado de los corredores ferroviarios. Sin embargo, en la toma de decisiones se debe analizar las posibles variables que puedan influir en el costo mínimo del trazado, esto lo sostiene Calderon et al. (2015) que el éxito de los resultados de la metodología depende específicamente de la correcta inclusión y definición de las variables.

\section{CONCLUSIONES}

La investigación cumplió el objetivo central propuesto, ya que presentó un modelo computacional basado en inteligencia geográfica para calcular el diseño de corredores de viabilidad ambiental para la propuesta del ferrocarril bioceánico.

Los resultados fueron positivos, a grande escala por ser la etapa inicial del planeamiento de la ferrovía bioceánica, el escenario de corredores ambientales priorizó las áreas naturales protegidas y las tierras indígenas y el escenario de los corredores no ambientales priorizó las variables de concesionarias mineras y agricultura; teniendo en cuenta estos condicionantes buscó soluciones evitar y atraer la interacción de la infraestructura con ellos. Como resultado de esta fase se obtienen una serie de soluciones, definidas como corredores o como líneas o trazados básicos. Todo ello sirve como punto de partida para el desarrollo del documento inicial de proyecto.

AGRADECIMIENTOS: A la Organización de Estados Americanos (OEA) y a la Coordenação de Aperfeiçoamento de Pessoal de Nível Superior - Brasil (CAPES) por la beca de estudio de maestría y al Conselho Nacional de Pesquisa e Desenvolvimento, Brasil (CNPq).

\section{REFERENCIAS}

ARCE, R.; ORTEGA, E.; OTERO, I.. Los Sistemas de Información Geográfica aplicados a la Evaluación Ambiental en la planificación de infraestructuras de transporte. Ciudad y Territorio: Estudios Territoriales, v.42, n.165-166, p.513528, 2010.

BARBOSA, P. G.; CAVALCANTI, A. B.; CAMPOS, J. H. B. C.; ROCHA, C. M. R. S.; SILVA, M. T.; OGATA, I. S.; COSTA, L. M.;
JUSTO, A. O.; CHAGAS, G. R.. Revista Ibero-Americana de Ciências Ambientais Uso de geotecnologias integradas a um sistema de Informações Geográficas ( SIG ) na análise da situação ambiental da região do parque estadual do Jabre / PB Use of geotechnologies integrated to a Geographic I. Revista Ibero Americana de Ciências Ambientais, v.10, n.6, p.146-153, 2019. 
CAILLAUX, J.; NOVAK, F.; RUIZ, M.. Las relaciones bilaterales entre el Perú y la República Popular de China. Lima: Sociedad Peruana de Derecho Ambiental, 2016.

CALDERÓN, R.; MEDINA, O.. Evaluación multicriterio como soporte para la selección de la mejor ruta de la vía ferrea entre Yopal y Bogotá. Universidad Distrital Francisco Jose de Caldas, 2015

CARRASCO, M.; SÁNCHEZ, A. S.. Evaluación de impacto ambiental Redacción y tramitación de documentos. Madrid: AENOR, 2010.

CELEMÍN, J. P.. El Proceso Analítico Jerárquico en el Marco de la Evaluación Multicriterio: Un Análisis Comparativo. Geografía y Sistemas de Información Geográfica (GEOSIG), v.6, n.6, p.47-63, 2014

CHEE, Y. E.. Principies Underpinning Bioddiversity offsets anda Guidance on their use. In: VAN DER REE, R.; SMITH, D. J.; GRILO, C.. Handbok of road Ecology. Chichester: Blackwey, 2015. p.51-64.

CREEG. China Railway Eryuan Engineering Group. Conexión Ferroviaria Bioceánica Perú/Brasil. Lima: CREEG, 2015.

CRAIG, N. B.. Estrada de Ferro Madeira: Mamoré. Companhia Editora Nacional, v.242, p.460, 1947.

DORADOR, W.. Interconexión Ferroviaria Bioceánica Perú Brasil: Análisis sobre la conveniencia del Proyecto. Tesis (Maestria en Diplomacia y Relaciones Exteriores) - Academia Diplomática del Perú “Javier Pérez de Cuellar”, Lima, 2016.

DOUROJEANNI, M.; BARANDIARÁN, A.; DOUROJEANNI, D.. Amazonía Peruana en 2021. Peru: ProNatural, 2009.

GONZÁLES, E.; MORENO, E.;CABELLO, F.; IVAREZ, E.. Metodología de trazado de corredores ferroviarios de mínimo impacto ambiental. Caso particular: Alta velocidad Huelva (España) - Faro (Portugal). Informes de la Construcción, v.64, n.527, p.415-423, 2012. DOI: https://doi.org/10.3989/ic.11.029

HERNÁNDEZ, K.. Relationship beteween Spatial Price Transmission and Geographical Distance. Agricultural and Applied Economics Association., 2011.

HOPEWELL, K.. New Protagonist in Global Economic Governance: Brazilian Agribusiness at the WTO*. Journal of Chemical Information and Modeling, v.8, n.9, p.1-58, 2017.

INEI. Directorio de Comunidades Nativas y Campesinas: Censo Nacional 2017. Lima, 2018.

MINAGRI. IV Trimestre 2018. Lima: MINAGRI, 2018.
MINEM. ANUARIO MINERO 2018. Lima: MINAGRI, 2018

NÓBREGA, R. A.; COSTA, A.; AZEVEDO, U.; TEIXEIRA, F.; SOUZA, I.; TISLER, T.; SOUZA, M.; PENA, M.. Quantificação da aderência do corredor de viabilidade calculado por análise multicritério e o traçado planejado da Ferrovia FICO. Belo Horizonte, 2017.

NÓBREGA, R. A.; VIEIRA, R. R.; BERBERIAN, C.; FILHO, N.; MASUKAWA, N.; FERRAZ, C. A.; QUADRO, E. A.. Inteligência geográfica para avaliação de propostas de projeto de concessão de corredores ferroviários. Transportes, v.24, n.4, p.1-10, 2016. DOI:

https://doi.org/10.14295/transportes.v24i4.1077

NÓBREGA, R. A.; STICH, B.; HOLLAND, J. H.; O'HARA, C.. Using multi-criteria decision making to highlight stakeholders' values in the corridor planning process. The Journal of Transport and Land Use, v.4, n.3, p.105-118, 2011. DOI: https://doi.org/10.5198/jtlu.v4i3.171

NOVAK, F.; NAMIHAS, S.. Las relaciones bilaterales entre el Perú y la República Popular de China. Sociedad Peruana de Derecho Ambiental, 2016.

PROACRE. Anexo 4: Marco Indigena. Rio Branco: PROACRE, 2012.

QUINTERO, J. D.. Carreteras ambientalmente amigables. Latin America Conservation Council, 2009

SAATY, T. L.; VARGAS, L. G.. The Seven Pillars of the Analytic Hierarchy Process. In: Models, Methods, Concepts \&amp; Applications of the Analytic Hierarchy Process. New York: Operations Research \& Management"Science, 2012. p.2340. DOI: https://doi.org/10.1007/978-1-4614-3597-6

SADASIVUNI, R.; CHARLES, O. H.. A Transportation corridor case study for multi-criteria decision Analysis. In: ASPRS ANNUAL CONFERENCE BALTIMORE, MARYLAND. Anais. Baltimore: Mississippi State University, 2009.

SERNAP. Memoria Anual 2017. Lima: SERNAP, 2018.

SOARES FILHO, B. S.; RODRIGUES, H. O.; COSTA, W. L.. Modeling environmental dynamics with Dinamica EGO. Instituto de Geociências - Centro de Sensoriamento Remoto, 2009.

VALEC. Relátorio Final Análise Multicriterial, identificação e Seleção da Alternativa de Traçado Lucas do Rio Verde/MT Vilhena/RO. Brasília: VALEC, 2014

WWF. World Wide Fund for Nature. Implementação da Avaliação Rápida e Priorização da Gestão de Unidades de Conservação Rappam em Unidades de Conservação no Estado do Acre. Brasília: WWF, 2010.

A CBPC - Companhia Brasileira de Produção Científica (CNPJ: 11.221.422/0001-03) detém os direitos materiais desta publicação. Os direitos referem-se à publicação do trabalho em qualquer parte do mundo, incluindo os direitos às renovações, expansões e disseminações da contribuição, bem como outros direitos subsidiários. Todos os trabalhos publicados eletronicamente poderão posteriormente ser publicados em coletâneas impressas sob coordenação da Sustenere Publishing, da Companhia Brasileira de Produção Científica e seus parceiros autorizados. Os (as) autores (as) preservam os direitos autorais, mas não têm permissão para a publicação da contribuição em outro meio, impresso ou digital, em português ou em tradução. 